\title{
Desarrollo del domino afectivo matemático en la formación inicial de maestros de primaria
}

\author{
José M. Marbán, Universidad de Valladolid (España) \\ Andrés Palacios, Universidad de Valladolid (España) \\ Ana Maroto, Universidad de Valladolid (España)
}

\section{Desarrollo del domino afectivo matemático en la formación inicial de maestros de primaria}

\section{Resumen}

Un elemento clave para mejorar cualquier sistema educativo es la formación inicial de maestros, donde en particular se construye el perfil docente bajo la influencia de factores afectivo-emocionales matemáticos. El objetivo de este trabajo es determinar si la formación universitaria produce cambios en el dominio afectivo matemático de los futuros maestros. Mediante un diseño longitudinal aplicado a 414 estudiantes del Grado en Educación Primaria y Modelos Lineales Generales de Medidas Repetidas, comparamos aspectos afectivo-emocionales al comienzo y al final de la formación. Los resultados indican la disminución de actitudes y emociones matemáticas positivas. Concluimos sobre la necesidad de 1) fijar mayor atención al dominio afectivo matemático en los programas de formación inicial de maestros y 2) desarrollar programas de intervención sobre este dominio en esta etapa formativa.

Palabras clave. Dominio afectivo; educación matemática; formación inicial; diseño longitudinal; futuros maestros de primaria.

\section{Development of primary school pre-service teachers' affective domain in mathematics}

\section{Abstract}

One key element to improve any educational system is pre-service teacher training, with the construction of the teaching profile particularly influenced by affective-emotional mathematical factors. The aim of this work is to determine whether university education produces changes in the mathematical affective domain of primary school pre-service teachers. By means of a longitudinal design applied to 414 students of the Primary Education Degree and General Linear Models of Repeated Measures, we compared affective-emotional aspects at the beginning and at the end of the training. Results indicate a decrease in positive mathematical attitudes and emotions. We conclude about the need of both: 1) paying greater attention to the affective domain in mathematics in pre-service teacher training programs and 2) developing intervention programs on this domain at such initial educational stage.

Keywords. Affective domain; mathematics education; teacher training; longitudinal study; primary school pre-service teachers.

\section{Introducción}

El interés por el estudio del dominio afectivo en la investigación en educación matemática ha aumentado de manera significativa en las últimas décadas (Jenßen, Dunekacke, Eid y Blömeke, 2015), siendo seminal el trabajo de McLeod (1989) donde se presentaba el dominio afectivo como una amalgama compleja de subdominios diferenciados de aspectos estrictamente cognitivos. Las obras de McLeod potenciaron el relanzamiento de las emociones en un campo usualmente caracterizado por estereotipos acerca de la supuesta objetividad de cálculos racionales. Así, surgieron nuevos desafíos y horizontes en los que las características afectivas adquirieron gran relevancia y el análisis de estas características se erigió como tema prioritario.

Se entiende el dominio afectivo matemático como un constructo formado por las creencias matemáticas, las emociones y las actitudes hacia esta materia. Las creencias 
están compuestas por elementos afectivos, evaluativos y sociales con una fuerte estabilidad. Este conocimiento se refiere a las matemáticas, a su enseñanza y a su aprendizaje. Las emociones, por otra parte, son rápidos cambios de sentimientos que aparecen como respuesta a un suceso interno o externo y que tienen un significado positivo o negativo para el individuo (Hidalgo, Maroto y Palacios, 2015).

Como tercer aspecto del dominio afectivo, definimos las actitudes como predisposición evaluativa (positiva o negativa) que condiciona las intenciones personales, con un fuerte componente corporal y gran influencia en el comportamiento. Cabe distinguir entre actitudes matemáticas y actitudes hacia las matemáticas. Las actitudes matemáticas están relacionadas con el modo y la manera de utilizar capacidades relevantes para el quehacer matemático (apertura mental, espíritu crítico, pensamiento reflexivo, etc.) y se relacionan más con lo cognitivo. Las actitudes hacia las matemáticas se refieren a la valoración, el aprecio y el gusto por esta disciplina y por su aprendizaje y reflejan más el componente afectivo que el cognitivo.

Además, en la formación inicial del maestro cabe considerar un tercer aspecto actitudinal: las actitudes hacia la enseñanza de las matemáticas, que siendo distintas están en relación con el resto de componentes emocionales. Los futuros docentes que, como estudiantes, tienen buenas actitudes hacia las matemáticas, también muestran actitudes positivas hacia la enseñanza de las matemáticas (Young-Loveridge, 2010).

La importancia de estos tres aspectos del dominio afectivo matemático parece evidente, siendo indudable que, en una dirección u otra, creencias, emociones y actitudes de los maestros van a influir en los aspectos cognitivos y afectivos de sus alumnos (Bailey, 2014). Las emociones de los futuros maestros van a tener una gran repercusión en la forma de afrontar su práctica docente (Jong, Hodges, Royal y Welder, 2015), en las relaciones interpersonales con sus alumnos y, todo ello, va a influir en los resultados de aprendizaje. Sakiz, Pape y Hoy (2012) han encontrado que un elemento importante para el desarrollo de los estudiantes en matemáticas es cómo sus maestros los han apoyado emocionalmente. Este apoyo determina su percepción sobre su capacidad, siendo un motor para su esfuerzo e, indirectamente, para su rendimiento escolar.

De todas las emociones, ha sido la ansiedad hacia las matemáticas de los futuros docentes una de las que ha recibido más atención desde la investigación. La razón de esto podría obedecer a los numerosos estudios que identifican la ansiedad matemática como fenómeno en los docentes en formación inicial (Hollingsworth y KnightMcKenna, 2018; Jacobs y Durandt, 2017). La presencia de esta emoción es especialmente relevante por su influencia negativa sobre las actitudes hacia las matemáticas y sobre su enseñanza. Bursal y Paznokas (2006) han sugerido que los futuros profesores con altos niveles de ansiedad tienden a confiar menos en su capacidad para enseñar Matemáticas. Mizala, Martínez y Martínez (2015) encontraron que la ansiedad puede influir negativamente en los futuros docentes en las expectativas de rendimiento sobre sus estudiantes y que esto podría afectar a su capacidad de desarrollar entornos inclusivos. Algunos autores han logrado relacionar la ansiedad de futuros maestros y la incapacidad para emprender procesos de enseñanza-aprendizaje de las matemáticas de calidad y eficientes (Boyd, Foster, Smith y Boyd, 2014), lo cual en muchos casos persiste a lo largo de la formación docente (Gresham, 2017).

Desde Fennema y Sherman (1976) se estudia el peso del gusto por las matemáticas en las actitudes. Recientemente, Palacios, Arias y Arias (2014) han encontrado este gusto como un factor principal de las actitudes. En futuros docentes se ha documentado que sus actitudes hacia las matemáticas influyen sobre su gusto por las matemáticas y 
por su enseñanza (Kunter et al., 2008). Khezri, Lavasania, Malahmadia y Amania (2010) vieron que los estudiantes con mayores percepciones de utilidad mostraban mayor deseo de estudiar matemáticas, así como mejores actitudes hacia las matemáticas y su enseñanza. Kim y Hodges (2012) obtuvieron correlaciones altas y significativas entre la percepción de utilidad de las matemáticas y la motivación para su estudio.

La percepción de utilidad es otro aspecto del dominio afectivo matemático. Khezri et al. (2010) concluyen que los estudiantes con un alto autoconcepto consideran que las matemáticas son útiles y de gran valor. Kim y Hodges (2012) obtuvieron correlaciones altas y significativas entre la percepción de utilidad de las matemáticas y la motivación para su estudio. Diferentes trabajos con futuros maestros (Nortes y Nortes, 2017; Nortes y Nortes, 2014) han obtenido correlaciones entre gusto y percepción de utilidad de modo que, aunque a los estudiantes no les gustan, las consideran una materia útil. En este mismo sentido, Gamboa y Moreira-Mora (2017) obtienen altas puntuaciones en cuanto a la percepción de utilidad de las matemáticas entre futuros maestros, pese a que estos mismos futuros maestros no consideran especialmente relevante su estudio. Tossavaine y Juvonen (2015) encuentran que la percepción de utilidad y el gusto por las matemáticas disminuyen a medida que lo hace el nivel educativo de los estudiantes.

Con respecto a la percepción de dificultad de las matemáticas como otro componente del dominio afectivo, esta percepción de dificultad aumenta a lo largo de la formación de los futuros maestros. Esta percepción de dificultad es para Hidalgo, Maroto y Palacios (2004) el antecedente del rechazo a la asignatura de matemáticas.

Estos trabajos muestran la importancia de estudiar el dominio afectivo matemático de maestros en formación. Su relación con la gestión y práctica docente del aula justifica el énfasis que debe hacerse en los programas de formación de maestros, con intervenciones de aula que refuercen los afectos hacia las matemáticas. Un adecuado programa puede modificar actitudes, creencias y emociones (Giles, Byrd y Bendolph, 2016; Van der Beek, Van der Ven, Kroesbergen y Leseman, 2017).

No obstante, son pocos los trabajos que han analizado la influencia que el proceso de formación de los futuros maestros tiene sobre estos aspectos afectivo-emocionales matemáticos. Sabemos que creencias, emociones y actitudes hacia las matemáticas sufren un lento pero continuo empeoramiento en una parte de los estudiantes. De modo que hay estudiantes que comienzan su formación como docentes con actitudes negativas hacia las matemáticas, con niveles no deseados de ansiedad y con creencias negativas sobre su utilidad o dificultad. Cabe suponer que la formación didáctica y los estudios del grado de maestro han de posibilitar la mejora de actitudes hacia las matemáticas, la disminución de niveles de ansiedad o la mejora en la percepción de su utilidad percibida. Pocos trabajos se plantean examinar la posibilidad de mejora.

Pretendemos determinar el grado de cambio en el dominio afectivo matemático, tras la formación de futuros maestros en centros universitarios con distintos planes de estudio. El objetivo es comparar los niveles de ansiedad, de las actitudes hacia las matemáticas y de su docencia, del gusto por esta materia, de la percepción de su utilidad y dificultad al comenzar la formación matemática universitaria y los valores al finalizar.

\section{Instrumentos y métodos}

Hemos trabajado con 414 estudiantes futuros docentes mediante un muestreo no probabilístico por accesibilidad entre los estudiantes de las universidades participantes. Para la recogida de datos se siguió a todos los participantes a lo largo del Grado de 
Educación Primaria (diseño longitudinal), con toma de datos en dos momentos: en los primeros cursos (toma inicial), dependiendo de la primera asignatura directamente vinculada con la Didáctica de la Matemática y siempre en las primeras semanas; en el tercer o cuarto curso y siempre en las últimas semanas antes de la finalización de la última asignatura relacionada directamente con la Didáctica de la Matemática. Esto se llevó a cabo durante los cursos escolares 2011-2012, 2012-2013, 2013-2014, 2014-2015, 2016-2017 y 2017-2018. El 18\% de estudiantes eran de primer curso en el momento de la toma de datos, el $31 \%$ de segundo, el $30 \%$ de tercero y el restante $21 \%$ de cuarto curso del grado. Los participantes cursaban la titulación en cuatro centros universitarios: $\mathrm{X}$ $(29,3 \%)$, Y $(21,3 \%), \mathrm{Z}(22,5 \%)$ y W (26,9\%). El $69 \%$ de los encuestados eran mujeres y el $31 \%$ hombres, distribución que no se diferencia de los valores medios de este tipo de estudios en España.

Tabla 1. Subescalas finales para toma de datos

\begin{tabular}{|c|c|c|c|}
\hline Escala & Ítems & Fuentes & $\begin{array}{c}\text { Alfa } \\
\text { C }\end{array}$ \\
\hline $\begin{array}{l}\text { ACTITUDES } \\
\text { HACIA } \\
\text { DOCENCIA } \\
\text { DE LA } \\
\text { MATEMÁTICA } \\
\text { (6 ítems) }\end{array}$ & $\begin{array}{l}\text { Me gusta ser profesor de matemáticas en primaria } \\
\text { Preferiría no tener que explicar matemáticas en mi futuro } \\
\text { ejercicio como maestro } \\
\text { Me siento cómodo explicando cómo he resuelto un problema } \\
\text { de matemáticas } \\
\text { Quiero ser un buen maestro, pero ique las matemáticas las } \\
\text { expliquen otros compañeros! } \\
\text { Si he elegido ser maestro es para poder explicar matemáticas } \\
\text { Me gusta más enseñar matemáticas que cualquier otra materia } \\
\text { del currículum de primaria }\end{array}$ & $\begin{array}{l}\text { McGinnis } \\
\text { et al. } \\
\text { (2002) }\end{array}$ & ,87 \\
\hline $\begin{array}{c}\text { ACTITUDES } \\
\text { HACIA } \\
\text { DIDÁCTICA } \\
\text { DE LA } \\
\text { MATEMÁTICA } \\
\text { (6 ítems) }\end{array}$ & $\begin{array}{l}\text { Para mi futuro profesional es fundamental entender las claves } \\
\text { de la enseñanza de las matemáticas } \\
\text { Si me lo propongo puedo entender las claves de la enseñanza } \\
\text { de las matemáticas } \\
\text { No es lo mismo saber matemáticas que saber enseñar } \\
\text { matemáticas. Esto segundo me gusta más } \\
\text { La didáctica de las matemáticas me acerca a las matemáticas } \\
\text { y me hace apreciar su enseñanza } \\
\text { La didáctica de las matemáticas me ayuda a entender las } \\
\text { matemáticas } \\
\text { La didáctica de las matemáticas me ha hecho valorar el } \\
\text { trabajo del profesor de matemáticas }\end{array}$ & $\begin{array}{c}\text { Fennema } \\
\text { y } \\
\text { Sherman } \\
(1976)\end{array}$ & ,81 \\
\hline $\begin{array}{c}\text { GUSTO POR LA } \\
\text { MATEMÁTICA } \\
\text { (7 ítems) }\end{array}$ & $\begin{array}{l}\text { Soy un aficionado a las curiosidades matemáticas } \\
\text { Me gustaría tener un conocimiento más profundo de las } \\
\text { matemáticas } \\
\text { Me gusta estudiar matemática en mi casa. } \\
\text { Si tuviera oportunidad me apuntaría a asignaturas optativas } \\
\text { relacionadas con las matemáticas } \\
\text { Me gusta tanto las matemáticas que me suelo inventar y } \\
\text { proponer nuevos problemas } \\
\text { Ha pedido el profesor de matemáticas voluntario para un } \\
\text { concurso de las matemáticas. iMe apuntaré! } \\
\text { Me gustan las matemáticas }\end{array}$ & $\begin{array}{c}\text { Fennema } \\
\text { y } \\
\text { Sherman } \\
(1976)\end{array}$ & ,83 \\
\hline $\begin{array}{c}\text { ANSIEDAD } \\
\text { HACIA LA } \\
\text { MATEMÁTICA } \\
\text { (7 ítems) }\end{array}$ & $\begin{array}{l}\text { Las matemáticas es una de las asignaturas que más temo } \\
\text { Las matemáticas hacen que me sienta incómodo y nervioso } \\
\text { Me dan miedo las matemáticas. }\end{array}$ & $\begin{array}{c}\text { Richardson } \\
\text { y Suinn } \\
\text { (1972) }\end{array}$ & ,94 \\
\hline
\end{tabular}




\begin{tabular}{|c|c|c|c|}
\hline & $\begin{array}{l}\text { Me angustio y siento miedo cuando el profesor me propone } \\
\text { por sorpresa que resuelva un problema de matemáticas } \\
\text { Cuando estudio matemáticas estoy más tenso que cuando lo } \\
\text { hago con otras asignaturas } \\
\text { Me siento generalmente inseguro cuando hago problemas de } \\
\text { matemáticas } \\
\text { En matemáticas sufro con frecuencia bloqueos }\end{array}$ & & \\
\hline $\begin{array}{c}\text { UTILIDAD } \\
\text { DE LA } \\
\text { MATEMÁTICA } \\
\text { (7 ítems) }\end{array}$ & $\begin{array}{l}\text { Las matemáticas son útiles y necesarias en todos los ámbitos } \\
\text { de la vida } \\
\text { Las matemáticas ayudan a entender el mundo de hoy } \\
\text { Las matemáticas pueden ser útiles para el que decida realizar } \\
\text { una carrera de Ciencias no para el resto } \\
\text { Tener buenos conocimientos de matemáticas incrementará } \\
\text { mis posibilidades de trabajo } \\
\text { Las matemáticas no sirven para nada } \\
\text { Para mi futuro profesional las matemáticas son una de las } \\
\text { asignaturas más importantes que tengo que estudiar } \\
\text { Una cierta comprensión de las matemáticas es hoy esencial }\end{array}$ & $\begin{array}{c}\text { Fennema } \\
\text { y } \\
\text { Sherman } \\
(1976)\end{array}$ & 80 \\
\hline $\begin{array}{l}\text { DIFICULTAD } \\
\text { DE LA } \\
\text { MATEMÁTICA } \\
\text { (6 ítems) }\end{array}$ & $\begin{array}{l}\text { Me resulta difícil comprender los conceptos matemáticos } \\
\text { Las matemáticas no son tan difíciles como dicen } \\
\text { Siempre he tenido problemas con las matemáticas } \\
\text { Las matemáticas son fáciles } \\
\text { Si las matemáticas fueran más sencillas, las estudiaría con } \\
\text { más interés } \\
\text { El mayor problema que veo en matemáticas es su dificultad }\end{array}$ & $\begin{array}{c}\text { Fennema } \\
\text { y } \\
\text { Sherman } \\
(1976)\end{array}$ & $\begin{array}{c}0,8 \\
3\end{array}$ \\
\hline
\end{tabular}

La toma de datos se realizó mediante una escala compuesta por seis subescalas para cuyo diseño y validación se partió, en una primera fase, de un amplio banco de ítems procedentes de diferentes escalas de validez y fiabilidad contrastada (ver Tabla 1). En todas las subescalas los ítems se enunciaban para ser respondidos según el grado de acuerdo con el correspondiente enunciado en una métrica tipo Likert de cinco puntos (valores de 0 a 4). Tras la adaptación al castellano, las subescalas iniciales se sometieron a un proceso de validación de contenido mediante juicio de expertos en Didáctica de la Matemática, atendiendo a cuestiones relacionadas con su relevancia (los ítems deberían estar relacionados con el objeto de estudio) y claridad (fácilmente comprensibles, con afirmaciones simples). Posteriormente, la versión de la escala resultante del mencionado proceso de validación fue sometida a diferentes pre-test con muestras reducidas de estudiantes que permitieron tanto garantizar su validez de apariencia como la selección de las preguntas o ítems que conformarían su versión final (Tabla 1).

El valor del coeficiente Alfa de Cronbach de la escala total fue de 0,95 (39 ítems); los valores de fiabilidad de las subescalas se resumen en la Tabla 1. Con las 6 subescalas se procedió a ejecutar un nuevo proceso de validez de contenido, esta vez mediante un Análisis Factorial Confirmatorio (AFC). Para su cálculo se utilizaron las matrices de covariaciones y el algoritmo de cálculo de los mínimos cuadrados no ponderados. El ajuste del modelo presenta valores adecuados (Tabla 2); además, la matriz LAMBDA$\mathrm{X}$ presenta todos los valores significativos con $\mathrm{p}>0,01$, lo que proporciona evidencias añadidas de la validez de las preguntas de las diferentes subescalas.

Tabla 2. Análisis Factorial Confirmatorio de la escala de dominio afectivo matemático

\begin{tabular}{ccccccc}
\hline $\begin{array}{c}\text { S-B } \\
(\text { Chi-cuadrado) } \\
(\mathrm{gl})(\mathrm{p})\end{array}$ & RMSEA & NFI & NNFI & CFI & AGFI & AIC \\
\hline $11961,40(968)$ &, 079 &, 96 &, 96 &, 97 &, 75 & 2162,00 \\
\hline
\end{tabular}


Los $\frac{(\mathrm{p}=0,00)}{\text { profesores colaboradores administraron las escalas, sin anonimato y con }}$ con consentimiento informado de los estudiantes. Las escalas y pruebas se realizaron en una sesión de hora y media. Los datos se analizaron mediante los paquetes estadísticos SPSS 23.0 (análisis descriptivos e inferenciales), Lisrel 8.7 (análisis factorial confirmatorio) y Statgraphics 16.2 (normalización de las variables). Las distribuciones de frecuencias se normalizaron cuando originariamente no lo estuvieran antes de realizar cálculo alguno. Para determinar la influencia del momento de toma de datos (variable independiente) sobre el rendimiento en las escalas (variables dependientes) se utilizaron Modelos Lineales Generales de Medidas Repetidas y subescalas. En este tipo de diseños, la variabilidad del análisis está presente a través de las diferencias entre los sujetos medidos en diferentes condiciones o momentos (diseños intra-sujetos). La significatividad estadística de los efectos se probó a través de la Traza de Pillai, siendo uno de los criterios más utilizados, junto con la Lambda de Wilks, la Traza de Hotelling y la Raíz mayor de Roy, para determinar la significación de los contrastes MANOVA, de los diseños intra-sujeto y de los longitudinales (Rodríguez-Jaume y Mora, 2001). El nivel de significación se ha mantenido para todos los cálculos en el $1 \%$.

\section{Resultados}

Los primeros análisis mostraron normalidad en los datos finales de 6 de las 12 variables involucradas. Antes de realizar los cálculos se normalizaron las puntuaciones de las escalas con distribuciones no normales. La Tabla 3 (con ' $M$ ' para Matemáticas) resume los valores descriptivos más relevantes, así como los resultados de las normalizaciones.

Tabla 3. Análisis descriptivo de resultados de las escalas originales y valores normalizados

\begin{tabular}{|c|c|c|c|c|c|c|c|c|c|c|}
\hline & \multicolumn{4}{|c|}{ Valores originales } & \multicolumn{4}{|c|}{ Valores normalizados } & \multicolumn{2}{|c|}{$\begin{array}{c}\text { Kolmogorov } \\
\text {-Smirnov }\end{array}$} \\
\hline & Media & D.T. & Asimetría & Curtosis & Media & D.T. & Asimetría & Curtosis & $\mathrm{Z}$ & Sig. \\
\hline $\begin{array}{l}\text { Actitudes } \\
\text { Docencia M } \\
\text { INICIAL }\end{array}$ & 11,90 & 4,64 &, 00 &,- 13 & $-{ }^{(*)}$ & - & - & - & 1,47 &, 03 \\
\hline FINAL & 12,06 & 5,00 &, 03 &,- 19 & $-^{(*)}$ & - & - & - & 1,43 &, 03 \\
\hline $\begin{array}{l}\text { Actitudes } \\
\text { Didáctica M } \\
\text { INICIAL }\end{array}$ & 14,81 & 4,02 &, 04 &,- 19 & $-\left(^{(*)}\right.$ & - & - & - & 1,60 &, 01 \\
\hline FINAL & 15,32 & 4,09 & ,21 &,- 31 & $-^{(*)}$ & - & - & - & 1,34 &, 05 \\
\hline $\begin{array}{l}\text { Gusto M } \\
\text { INICIAL }\end{array}$ & 10,35 & 4,44 &, 72 & ,99 & 10,48 & 4,61 & ,09 &,- 06 & 1,25 & ,09 \\
\hline FINAL & 10,42 & 4,65 &, 83 & 1,23 & 10,48 & 4,61 & ,09 &,- 06 & 1,13 &, 16 \\
\hline $\begin{array}{l}\text { Ansiedad M } \\
\text { INICIAL }\end{array}$ & 11,57 & 6,09 &, 58 &,- 05 & 11,62 & 6,01 &, 06 &,- 20 & 1,28 &, 07 \\
\hline FINAL & 11,97 & 6,77 & ,61 &,- 09 & 12,04 & 6,65 &, 03 &,- 33 & 1,35 &, 05 \\
\hline $\begin{array}{l}\text { Percepción } \\
\text { Utilidad M } \\
\text { INICIAL }\end{array}$ & 19,13 & 3,99 &,- 10 &,- 48 & $--^{(*)}$ & - & - & - & 1,15 &, 14 \\
\hline FINAL & 19,21 & 4,12 &,- 10 &,- 59 & $-^{(*)}$ & - & - & - & 1,50 &, 02 \\
\hline $\begin{array}{l}\text { Percepción } \\
\text { Dificultad M } \\
\text { INICIAL }\end{array}$ & 11,67 & 4,02 &, 25 &, 29 & $-^{(*)}$ & - & - & - & 1,42 &, 03 \\
\hline FINAL & 12,12 & 4,28 & , 18 &,- 10 & $-^{(*)}$ & - & - & - & 1,09 & ,19 \\
\hline
\end{tabular}

(*) Variables donde no fue necesaria la normalización 
Tras la normalización de todas las variables, se calcularon los valores medios de las diferentes subescalas al comienzo de la formación (datos iniciales) y a su finalización (datos finales) y se analizaron la significatividad estadística de la diferencia de medias en una y otra toma, cuyos resultados resumimos en la Tabla 4 (con E para "Escala").

Tabla 4. Diferencias de medias al comenzar y al finalizar la formación

\begin{tabular}{|c|c|c|c|c|c|c|c|c|}
\hline & \multicolumn{2}{|c|}{ Datos iniciales } & \multicolumn{2}{|c|}{ Datos finales } & \multicolumn{4}{|c|}{ MLG de Medidas Repetidas } \\
\hline & Media & D.T. & Media & D.T. & $\begin{array}{l}\text { Traza } \\
\text { Pillai }\end{array}$ & $\mathrm{F}$ & sig & $\begin{array}{l}\text { Potencia } \\
\text { observada }\end{array}$ \\
\hline $\begin{array}{l}\text { E Actitudes } \\
\text { Docencia M }\end{array}$ & 11,85 & 4,85 & 12,02 & 5,00 &, 00 &, 59 & ,44 &, 04 \\
\hline $\begin{array}{l}\text { E Actitudes } \\
\text { Didáctica M }\end{array}$ & 14,61 & 4,20 & 15,30 & 4,12 & ,03 & 7,93 &, 01 &, 59 \\
\hline Gusto M & 10,30 & 4,20 & 10,42 & 4,57 & ,00 & ,28 & 60 & ,02 \\
\hline Ansiedad M & 11,58 & 6,07 & 12,05 & 6,65 &, 01 & 2,98 & ,09 & ,20 \\
\hline $\begin{array}{l}\text { Percepción } \\
\text { Dificultad M }\end{array}$ & 11,68 & 4,03 & 12,14 & 4,27 &, 02 & 4,67 & ,03 &, 34 \\
\hline $\begin{array}{l}\text { Percepción } \\
\text { Utilidad M }\end{array}$ & 19,24 & 3,89 & 19,21 & 4,12 &, 00 &, 01 & ,90 &, 01 \\
\hline
\end{tabular}

Los valores de la Traza de Pillai presentan diferencias significativas en una única variable: la Escala de Actitudes hacia la Didáctica de las Matemáticas. Se produce un aumento en los valores medios al finalizar la formación matemática mejorando las actitudes hacia el valor educativo y formativo de la Didáctica de las Matemáticas. El análisis de los valores medios de los seis ítems de esta subescala de Actitudes hacia la Didáctica de las Matemáticas al comenzar y al terminar la formación del Grado denota que dicha formación ha influido positivamente en el valor que los futuros maestros otorgan a la materia (Tabla 5). Al finalizar su formación, los estudiantes dicen comprender el papel de entender las claves de la enseñanza de las matemáticas para su futuro profesional en mayor medida que al inicio $(\mathrm{F}=11,68 ; \mathrm{p}>, 01)$. Afirman distinguir con valores medios mayores al final de su formación lo que significa saber matemáticas de saber enseñar matemáticas $(\mathrm{F}=8,40 ; \mathrm{p}>, 01)$ y consideran más positivamente el papel de la Didáctica en la valoración del docente de matemáticas $(F=9,99 ; p>, 01)$.

Tabla 5. Valores medios al comenzar y al finalizar la formación (Escala de Actitudes hacia la Didáctica de las Matemáticas)

Datos iniciales Datos finales MLG de Medidas Repetidas

\begin{tabular}{|c|c|c|c|c|c|c|c|c|}
\hline & Media* & D.T. & Media(*) & D.T. & $\begin{array}{l}\text { Traza } \\
\text { Pillai } \\
\end{array}$ & $\mathrm{F}$ & Sig. & Pot. \\
\hline $\begin{array}{l}\text { Para mi futuro profesional es } \\
\text { fundamental entender las claves de } \\
\text { la enseñanza de las matemáticas }\end{array}$ & 2,77 & 1,00 & 2,98 & ,93 & ,04 & 11,68 & ,00 & ,93 \\
\hline $\begin{array}{l}\text { Si me lo propongo puedo entender } \\
\text { las claves de la enseñanza de las } \\
\text { matemáticas }\end{array}$ & 2,75 & ,86 & 2,76 & ,90 & ,00 & ,61 & ,43 &, 12 \\
\hline $\begin{array}{l}\text { No es lo mismo saber matemáticas } \\
\text { que saber enseñar matemáticas. Estc } \\
\text { segundo me gusta más }\end{array}$ & 2,55 & ,98 & 2,60 & 1,02 & ,01 & 8,40 & ,00 & ,45 \\
\hline $\begin{array}{l}\text { La didáctica de las matemáticas me } \\
\text { acerca a las matemáticas y me hace } \\
\text { apreciar su enseñanza }\end{array}$ & 2,23 & ,86 & 2,28 & ,93 & ,00 & 1,18 & .28 & , 19 \\
\hline $\begin{array}{l}\text { La didáctica de las matemáticas me } \\
\text { ayuda a entender las matemáticas }\end{array}$ & 2,33 &, 87 & 2,39 & ,98 & ,00 & ,09 &, 77 &, 06 \\
\hline
\end{tabular}


La didáctica de las matemáticas me ha hecho valorar el trabajo de profesor de matemáticas

2,37

,91

2,52

, 93

, 04

9,99

, 00 8

(*) Valores medios de una escala de 0 a 4 puntos

No hay diferencias significativas en los valores medios del resto de subescalas. En la escala de Actitudes hacia la Docencia de las Matemáticas, los estudiantes terminan sus estudios con niveles actitudinales parecidos a los que tenían cuando empezaron. Incluso el deseo de impartir matemáticas en Primaria disminuye en el paso de los primeros a los últimos cursos; así, un 39,5\% de los estudiantes al comienzo de su formación manifestaban deseos de impartir matemáticas, porcentaje que baja hasta el $33,40 \%$ al final de la formación. Además, estos mismos estudiantes al final de su formación manifiestan deseos de no impartir docencia de matemáticas en un 68,30\%, porcentaje que al comienzo de la formación suponía el $61,30 \%$ de los estudiantes. Resultados similares se obtienen en la escala de Gusto por las Matemáticas, aunque como hemos indicado las diferencias no son significativas. Cabe resaltar que el número de estudiantes que manifestaban al comienzo de su formación que les gustaban las matemáticas era de un 25,30\%; este mismo dato baja al finalizar su formación hasta un $21,80 \%$.

Con respecto a la Percepción de Utilidad de las Matemáticas y a la Percepción de Dificultad de las Matemáticas, al término de su formación, una parte importante de estudiantes consideran las matemáticas útiles $(70,2 \%)$ con valores parecidos a cuando comenzaron $(68,90 \%)$; además, siguen considerando esta materia difícil al inicio $(25,10 \%)$ y al final de la de formación $(31,80 \%)$. Los estudiantes que al comenzar su formación opinaban que les costaba comprender conceptos matemáticos $(14,30 \%)$, apenas modifican su opinión al finalizar $(16,30)$. La Ansiedad hacia las Matemáticas está igualmente presente al comenzar la formación y al finalizar. Una parte significativa de los estudiantes noveles manifiestan tener miedo a las matemáticas $(16,00 \%)$, cifra que sube ligeramente al final de su formación $(17,30 \%)$. La vivencia de temor se da en porcentajes parecidos tanto al inicio $(29,00 \%)$ como al final $(27,30 \%)$. Los bloqueos mentales asociados a las matemáticas son descritos por un porcentaje parecido entre los estudiantes noveles $(23,80 \%)$ y los que cursan últimos cursos $(25,50 \%)$.

Tabla 6.- Diferencia en valores medios por clúster (escalas afectivo emocional matemático)

\begin{tabular}{lccccccc}
\hline & & \multicolumn{6}{c}{ Escalas } \\
\cline { 3 - 8 } & & E Actitudes & E Actitudes & Gusto & Ansiedad & Percepción & Percepción \\
Clúster & & Docencia M & Didáctica M. & M & M & Dificultad M. & Utilidad M. \\
\hline \multirow{2}{*}{1} & Media & 12,81 & 14,75 & 11,31 & 10,22 & 10,99 & 18,77 \\
& D.T. & 3,24 & 3,81 & 2,92 & 3,09 & 2,32 & 3,79 \\
2 & Media & 17,52 & 17,50 & 15,05 & 3,92 & 6,71 & 22,20 \\
& D.T. & 3,31 & 3,98 & 3,06 & 3,72 & 2,26 & 3,04 \\
3 & Media & 7,78 & 13,43 & 6,73 & 17,67 & 15,23 & 18,43 \\
Significación & D.T. & 3,87 & 4,16 & 2,98 & 3,73 & 3,15 & 3,67 \\
diferencia & F & 156,61 & 19,69 & 161,96 & 326,60 & 207,75 & 22,71 \\
medias & Sig. &, 00 &, 00 &, 00 &, 00 &, 00 &, 00 \\
\hline
\end{tabular}

Si bien no es objeto de este trabajo, se ha valorado incorporar, como complemento a los análisis anteriores, un análisis de clúster para establecer tipologías de estudiantes al comenzar su formación a partir de los datos de las seis variables del estudio. Para la asignación de los sujetos a cada grupo uno de los sistemas más utilizado es el conocido como método de clasificación no jerárquico (análisis de conglomerados de k-medias). Aunque en nuestro caso existen varias soluciones posibles, nos hemos decantado por tres clústeres dado que esta solución maximiza la varianza entre tipologías y minimiza 
la varianza dentro de cada clúster, criterio este que suele utilizarse para determinar el número de grupos de manera estándar (Palacios y López-Pastor, 2013). El primero de estos conglomerados está formado por 154 estudiantes $(47,83 \%)$, el segundo por 56 $(17,39 \%)$ y el tercero por $112(34,78 \%)$, quedando fuera de los conglomerados aquellos casos con valores perdidos. Para caracterizar cada uno de estos grupos se han calculado los valores medios de cada clúster, en cada una de las escalas (Tabla 6).

El Clúster 2, el que está formado por un menor número de estudiantes (17,34\%), tiene los valores medios más elevados en las Actitudes hacia Docencia de la Matemática, las Actitudes hacia Didáctica de la Matemática, el Gusto por la Matemática y la Utilidad de la Matemática y los valores menores en la escala de Ansiedad y en la de Percepción de Dificultad. El Clúster 3, formado por algo más de un tercio de la muestra $(34,78 \%)$, tiene las medias más bajas en las Actitudes hacia Docencia de la Matemática, las Actitudes hacia Didáctica de la Matemática y el Gusto por la Matemática y las medias más elevadas en Ansiedad y en Percepción de Dificultad. Por último, el Clúster 1, el más numeroso con un 47, 83\%, presenta valores intermedios en todas las escalas. Es interesante señalar que son escasos los cambios que se han producido en cada segmento a lo largo de la formación (Tabla 7). Las correlaciones entre las puntuaciones de las 6 escalas al inicio y al final, en cada uno de los conglomerados, son todas positivas y estadísticamente significativas en la mayoría de los casos.

Tabla 7. Correlaciones al comienzo y al final de la formación en cada uno de los clúster

\begin{tabular}{|c|c|c|c|c|c|c|}
\hline & \multicolumn{6}{|c|}{ Escalas (inicial vs final) } \\
\hline & $\begin{array}{l}\text { Actitudes } \\
\text { Docencia } \\
\end{array}$ & $\begin{array}{l}\text { Actitudes } \\
\text { Didáctica } \\
\end{array}$ & Gusto & Ansiedad & $\begin{array}{c}\text { Percepción } \\
\text { Dificultad } \\
\end{array}$ & $\begin{array}{c}\text { Percepción } \\
\text { Utilidad }\end{array}$ \\
\hline $\begin{array}{l}\text { Clúster } 1 \\
\text { Perfil "positivo" }\end{array}$ &, $48^{* *}$ &, $33^{* *}$ &, $33^{* *}$ & $48^{* *}$ &, $40^{* *}$ & $25^{* *}$ \\
\hline $\begin{array}{l}\text { Clúster } 2 \\
\text { Perfil neutro }\end{array}$ &, $46^{* *}$ & $63^{* *}$ &, $58^{* *}$ &, 18 &, 06 & $27^{* *}$ \\
\hline $\begin{array}{l}\text { Clúster } 3 \\
\text { Perfil "negativo" }\end{array}$ &, $55^{* *}$ &, $56^{* *}$ &, 14 & $47^{* *}$ &, $36^{* *}$ &, $37^{* *}$ \\
\hline
\end{tabular}

\section{Discusión y conclusiones}

Una primera conclusión apunta a un estancamiento de actitudes, emociones y creencias en el paso por la formación docente de futuros maestros de matemáticas, como se desprende de la ausencia de diferencias significativas en el inicio y el final de la formación en 5 de las 6 escalas utilizadas. Así, al finalizar su formación universitaria, los estudiantes no han reducido sus miedos, no han cambiado sus creencias sobre las matemáticas ni han mejorado sus actitudes hacia la materia. Este hecho es especialmente relevante si tenemos en cuenta que, sobre el papel, el estudio realizado sirve como contraste de hasta qué punto modelos de enseñanza y aprendizaje centrados en el estudiante pueden actuar como catalizadores de cambios positivos en el dominio afectivo matemático. Esta hipótesis parte de la consideración a priori de que ciertas reformas educativas incluyen entre sus principios la necesidad de involucrar activamente a los maestros en formación. El tipo de situaciones en las que el estudiante hace suyo el problema y genera la necesidad de resolverlo, el interés por resolverlo o bien ambas cuestiones, le acercan a la disciplina y a sus aplicaciones prácticas. A su vez, cabría esperar que este acercamiento entre realidad individual y objeto de estudio provocase efectos positivos en el ámbito del dominio afectivo. Los resultados, sin embargo, no apuntan en esta dirección, sin que sea posible concluir a partir de los datos disponibles 
si las discrepancias se hallan en una incompleta o meramente formal implantación de modelos de enseñanza y aprendizaje que se ajusten a lo pretendido o en la incapacidad por sí solos de alterar significativamente estadios tan resistentes al cambio como los que afectan al dominio afectivo. Si bien no se descarta que la primera de las dos causas mencionadas juegue su papel en la interpretación de los resultados, es evidente, a la luz de estudios como los de Beilock, Gunderson, Ramirez y Levine (2010), que elementos como el diseño curricular, el método docente o la motivación no son suficientes para modificar, por si solos, el perfil afectivo-emocional del estudiante.

Los resultados, por tanto, no permiten una interpretación única. Hemos de tener en cuenta que desarrollar competencia matemática, siendo una tarea compleja parece ser un objetivo más fácil que el de cambiar el perfil emocional matemático. Adquiere así sentido la idea de fuerza de las actitudes con capacidad para permanecer estables en el tiempo y resistentes al cambio, frente a las actitudes que serían débiles. La congruencia de contenidos (cognitivo, emocional y conductual) y la duración en el tiempo son dos elementos que definirían dicha fuerza. Las actitudes hacia las matemáticas tienden a poseer ambos rasgos: son permanentes a lo largo del tiempo (Hidalgo, Maroto y Palacios, 2015) y poseen un alto grado de consistencia en sus componentes (Hidalgo, Maroto y Palacios, 2004). Además, la consolidación de la valencia e intensidad de las actitudes se realiza tempranamente en la escuela (Jacobs y Durandt, 2017).

Si el perfil emocional matemático se consolida tempranamente, entonces un porcentaje significativo de futuros docentes comienzan su formación como maestros con una carga emocional matemática negativa arraigada (en nuestros datos con un porcentaje entre $16^{\prime} 00 \%$ y $17^{\prime} 30 \%$, cercanos a los obtenidos por Çatlioğlu, Birgin, Coştu y Gürbüz, 2009 , del $16^{\prime} 00 \%$ al $20{ }^{\prime} 00 \%$ ). En este contexto, no parece sencilla la tarea de modificar estas actitudes, creencias y emociones con un número de créditos de formación didáctico-matemática en el Grado de Primaria que oscila entre los 18 y los 24 créditos ECTS, valores que parecen revelarse como insuficientes para abordar con garantías de éxito el fin mencionado. En este sentido, conviene volver la mirada al concepto de competencia matemática y al de conocimiento profesional docente, atendiendo así al doble rol que los estudiantes para maestro de Primaria juegan durante su formación en el ámbito didáctico-matemático. Modelos como el de Ball, Lubienski y Mewborn (2001) aluden en su dominio relativo al conocimiento de los estudiantes a cuestiones afectivas, que pueden tener incidencia en los resultados académicos y en el devenir del proceso de enseñanza-aprendizaje. Sin embargo, una mirada a guías docentes y a directrices normativas de planes de estudio, basta para constatar que sobre el papel estos temas no son considerados explícitamente por lo que su reconocimiento teórico como variables relevantes no se traduce aparentemente en una implementación de aula.

Todos los argumentos anteriores apuntan a la necesidad de establecer programas de intervención afectivo-matemáticos específicos que acompañen los procesos de formación didáctica de los estudiantes para maestro. Giles et al. (2016) y Van der Beek et al. (2017) se refieren a experiencias sobre la eficacia de este tipo de programas para incidir en aspectos no cognitivos de la formación matemática. Son programas que deberían atender la especificidad del individuo y la colectividad del grupo en un enfoque constructivista que considere que los conflictos socio-afectivos en matemáticas son necesarios para el desarrollo de una buena competencia en el ámbito de la enseñanza y del aprendizaje de matemáticas. Una educación emocional orientada a un mejor conocimiento de las emociones propias y de las de los demás permitiría desarrollar la habilidad de regular las emociones, prevenir los efectos de las emociones negativas y desarrollar la habilidad para generar emociones positivas. 
Esta necesaria intervención en el aula marca una línea futura de investigación. Otras líneas futuras tienen que ver con las limitaciones de nuestra aportación. Es posible considerar los efectos de las variables "docente universitario" y "centro" en la existencia de diferencias significativas que pueden estar ocultas en los datos globales. No hemos diferenciado los programas de formación de maestros a la hora de considerar la muestra. Cabe señalar en este sentido, sin embargo, que, de acuerdo con Cebolla-Boado y Garrido-Medina (2013), España dispone de un sistema de alta equidad inter-centros, con un $2 \%$ de la varianza total del conocimiento matemático de los futuros maestros ligada a la agrupación en facultades. Una profundización en este tema permitiría determinar los efectos de la metodología didáctica, de los contenidos desarrollados y, en general, de la dinámica de cada aula y de cada centro universitario sobre los cambios en estos aspectos no cognitivos matemáticos en la formación inicial del maestro.

Siguiendo con el presente estudio, cabe señalar que el enfoque metodológico ha sido puramente cuantitativo, con uso de escalas construidas ad hoc y análisis mediante Modelos Lineales Generales de Medidas Repetidas. Así se ha llegado a una amplia muestra y el análisis ha sido relativamente fácil. No obstante, la lengua en las escalas puede ser fuente de sesgo e influir en las respuestas y, por otra parte, los datos informan en términos de visión de conjunto sin detalle individual, social ni cultural. Convendrá complementar este estudio con otros de corte más cualitativo que ayuden a entender y caracterizar el tema de los afectos. En este sentido, marcamos como línea de continuidad el acercamiento al perfil afectivo de los futuros maestros mediante el uso de instrumentos que les permitan hablar sobre lo que sienten hacia las matemáticas y su enseñanza, siendo la entrevista una opción. El interés de esta línea viene también avalado cuantitativamente por los resultados del análisis de conglomerados realizado en este trabajo, a través del cual se han identificado tres tipologías de estudiantes al comenzar su formación a partir de los datos de las 6 variables del estudio. Este análisis ha permitido comprobar, mediante análisis de correlaciones al comienzo y al final de la formación, que la "radiografía" se mantiene al finalizar la formación en cada uno de los clústeres. No ha sido posible incorporar en estos análisis el factor que da cuenta de la vocación (inicial o no) del estudiante en términos de especialización a través de una u otra mención cualificadora al no disponer de información. Esta es una información de interés en futuros estudios junto con otras que permitan caracterizar perfiles afectivoemocionales iniciales de estudiantes de cara a facilitar la identificación de diferencias individuales que permitan diseñar propuestas de actuación, apoyo y acompañamiento.

\section{Referencias}

Bailey, J. (2014). Mathematical investigations for supporting pre-service primary teachers. Repeating a mathematics education course. Australian Journal of Teacher Education, 39(2), 86-100.

Ball, D. L., Lubienski, S. T. y Mewborn, D. S. (2001). Research on teaching mathematics: The unsolved problem of teachers' mathematical knowledge. En V. Richardson (Ed.), Handbook of research on teaching (pp. 433-456). Washington DC: AERA.

Beilock, S. L., Gunderson, E. A., Ramirez, G. y Levine, S. C. (2010). Female teachers' math anxiety affects girls' math achievement. Proceedings of the National Academy of Sciences, 107(5), 1860-1863.

Boyd, W., Foster, A., Smith, J. y Boyd, W. E. (2014). Feeling good about teaching mathematics: Addressing anxiety amongst pre-service teachers. Creative Education, 5, 207-217. 
Bursal, M. y Paznokas, L. (2006). Mathematics anxiety and preservice elementary teachers' confidence to teach mathematics and science. School Science and Mathematics, 106(4), 173-180.

Çatlioğlu, H., Birgin, O., Coştu, S. y Gürbüz, R. (2009). The level of mathematics anxiety among pre-service elementary school teachers. Procedia-Social and Behavioral Sciences, 1(1), 1578-1581.

Cebolla-Boado, H. y Garrido-Medina, L. (2013). Los efectos de la educación universitaria en el conocimiento en matemáticas en España y en EE.UU: Evidencias del cuestionario TEDS-M. Informe español, Vol. II. Madrid: MEC.

Fennema, E. y Sherman, J. A. (1976). Fennema-Sherman mathematics attitudes scales: Instruments designed to measure attitudes toward the learning of mathematics by females and males. Journal for Research in Mathematics Education, 7(5), 324-326.

Gamboa, R. y Moreira-Mora, T. E. (2017). Actitudes y creencias hacia las matemáticas: un estudio comparativo entre estudiantes y profesores. Revista Electrónica Actualidades Investigativas en Educación, 17(1), 1-45.

Giles R. M., Byrd, K. O. y Bendolph, A. (2016). An investigation of elementary preservice teachers' self-efficacy for teaching mathematics. Cogent Education, 3, 111.

Gresham, G. (2017). Preservice to inservice: Does mathematics anxiety change with teaching experience? Journal of Teacher Education, 69(1), 90-107.

Hidalgo, S., Maroto, A. y Palacios, A. (2004). ¿Por qué se rechazan las matemáticas? Análisis evolutivo y multivariante de actitudes relevantes hacia las matemáticas. Revista de Educación, 334, 75-98.

Hidalgo, S., Maroto, A. y Palacios, A. (2015). Una aproximación al sistema de creencias matemáticas en futuros maestros. Educación Matemática, 27(1), 65-90.

Hollingsworth, H. L. y Knight-McKenna, M. (2018). "I am now confident": Academic service-learning as a context for addressing math anxiety in preservice teachers. Journal of Early Childhood Teacher Education, 39(4), 312-327.

Jacobs, G. J. y Durandt, R. (2017). Attitudes of pre-service mathematics teachers towards modelling: A South African inquiry. EURASIA Journal of Mathematics Science and Technology Education, 13(1), 61-84.

Jenßen, L., Dunekacke, S., Eid, M. y Blömeke, S. (2015). The relationship of mathematical competence and mathematics anxiety. Zeitschrift für Psychologie, 223, 31-38.

Jong, C., Hodges, T. E., Royal, K. D. y Welder, R. M. (2015): Instruments to measure elementary preservice teachers' conceptions. Educational Research Quarterly, 39(1), 21-48.

Khezri, H. Lavasania, M. G., Malahmadia, E. y Amania, J. (2010). The role of selfefficacy, task value, and achievement goals in predicting learning approaches and mathematics achievement. Procedia Social and Behavioral Sciences, 5, 942-947.

Kim, C. y Hodges, C. B. (2012). Effects of an emotion control treatment on academic emotions, motivation and achievement in an online mathematics course. Instructional Science, 40(1), 173-192.

Kunter, M., Tsai, Y.-M., Klusmann, U., Brunner, M., Krauss, S. y Baumert, J. (2008). Students' and mathematics teachers' perceptions of teacher enthusiasm and instruction. Learning and Instruction, 18, 468-482. 
McGinnis, J. R., Kramer, S., Shama, G., Graeber, A. O., Parker, C. A. y Watanabe, T. (2002). Undergraduates' attitudes and beliefs about subject matter and pedagogy measured periodically in a reform based mathematics and science teacher preparation program. Journal of Research in Science Teaching, 39(8), 713-737.

McLeod, D. B. (1989). Beliefs, attitudes, and emotions: New views of affect in mathematics education. En D. B. McLeod y V. M. Adams (Eds.), Affect and mathematical problem solving (pp. 245-258). Nueva York: Springer.

Mizala, A., Martínez, F. y Martínez, S. (2015). Pre-service elementary school teachers' expectations about student performance: How their beliefs are affected by their mathematics anxiety and student's gender. Teaching and Teacher Education, 50, 7078.

Nortes, R. y Nortes, A. (2014). Ansiedad hacia las matemáticas, agrado y utilidad en futuros maestros. En M. T. González, M. Codes, D. Arnau y T. Ortega (Eds.), Investigación en Educación Matemática XVIII (pp. 485-492). Salamanca: SEIEM.

Nortes, R. y Nortes, A. (2017). Agrado y utilidad de las matemáticas en la formación inicial de maestros de educación primaria. PNA, 12(1), 27-44.

Palacios, A., Arias, V. y Arias, B. (2014). Attitudes towards mathematics: Construction and validation of a measurement instrument. Revista de Psicodidáctica, 19(1), 6791.

Palacios, A. y López-Pastor, V. M. (2013). Haz lo que yo digo pero no lo que yo hago. Revista de Educación, 361, 279-305

Richardson, F. C. y Suinn, R. M. (1972). The mathematics anxiety rating scale: psychometric data. Journal of Counseling Psychology, 19(6), 551-554.

Rodríguez-Jaume, M. J. y Mora, R. (2001). Estadística informática: Casos y ejemplos con el SPSS. Alacant: Servicio de Publicaciones de la Universidad.

Sakiz, G., Pape, S. J. y Hoy, A. W. (2012). Does perceived teacher affective support matter for middle school students in mathematics classrooms? Journal of School Psychology, 50(2), 235-255.

Tossavainen, T. y Juvonen, A. (2015). Finnish primary and secondary students' interest in music and mathematics relating to enjoyment of the subject and perception of the importance and usefulness of the subject. Research Studies in Music Education, 37(1), 107-121.

Van der Beek, J. P., Van der Ven, S., Kroesbergen, E. y Leseman, P. (2017). Selfconcept mediates the relation between achievement and emotions in mathematics. British Journal of Educational Psychology, 87, 478-495.

Young-Loveridge, J. (2010). Two decades of mathematics education reform in New Zealand: What impact on the attitudes of teacher education students? Mathematics Education Research Journal, 33, 3-7.

\section{Referencias de los autores}

José M. Marbán, Universidad de Valladolid, josemaria.marban@uva.es

Andrés Palacios, Universidad de Valladolid, palacios@psi.uva.es

Ana Maroto, Universidad de Valladolid, anaisabel.maroto@uva.es 


\section{Development of school primary pre-service teachers' affective domain in mathematics}

José M. Marbán, Universidad de Valladolid

Andrés Palacios, Universidad de Valladolid

Ana Maroto, Universidad de Valladolid

The implementation of the European Higher Education Area meant a change in the organization of the university system and programs with a commitment to methods for active participation of students aimed at the development of mathematical and didacticmathematical skills, both cognitive and emotional. In the training of future mathematics teachers, any improvement deals with the identification and understanding of affective factors that determine features of the teaching profile. The goal of our research work with primary school pre-service teachers is to examine the influence of changes in their affective domain in mathematics. We carried out a longitudinal design with 414 students across their four-year Degree in Primary Education from four Spanish universities. All participants were followed throughout the Degree and measures were taken at two punctual times. The initial taking corresponded to the moment when students joined their first content subject on mathematics (education) and always during the first weeks of the course. The final taking was performed in the third or fourth academic year and always in the last weeks of the last mathematics (education) course. We used six subscales for measuring features of the affective domain in mathematics, all of them validated and with high reliability parameters. To determine the existence of significant differences, General Linear Models of Repeated Measures (intra-subject model) were applied in comparing results at the beginning and at the end of the teacher training process. Statistically significant differences were then found in the results provided by the Attitudes Towards Mathematics Teaching Scale only. These results show a stagnation or decrease in attitudes, emotions and positive mathematical beliefs in relation to the effects of teacher training in mathematics (education) over students. Our conclusions show the need of greater interest on the matters related to the affective domain in mathematics at the stage of initial training programs and of proposals of affective mathematical intervention programs that accompany the training processes. 